\title{
Relationship between Uric Acid and Subtle Cognitive Dysfunction in Chronic Kidney Disease
}

\author{
Baris Afsar $^{\mathrm{a}}$ Rengin Elsurer $^{\mathrm{a}}$ Adrian Covic $^{\mathrm{c}}$ Richard J. Johnson ${ }^{\mathrm{d}}$ \\ Mehmet Kanbay ${ }^{b}$ \\ ${ }^{a}$ Division of Nephrology, Department of Medicine, Zonguldak State Hospital, Zonguldak, ${ }^{b}$ Division of Nephrology,

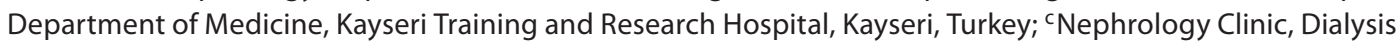 \\ and Renal Transplant Center, C.I. PARHON University Hospital, Gr. T. Popa University of Medicine and Pharmacy, \\ lasi, Romania; ${ }^{\mathrm{d}}$ Division of Renal Diseases and Hypertension, University of Colorado Denver, Denver, Colo., USA
}

\section{Key Words}

Cognitive function $\cdot$ Chronic kidney disease $\cdot$ Uric acid

\begin{abstract}
Background: Elevated serum uric acid has been associated with cognitive dysfunction and vascular cognitive impairment in the elderly. Serum uric acid is also commonly elevated in chronic kidney disease (CKD), but its relationship with cognitive function in these patients has not been addressed. Methods: Subjects with CKD (defined as eGFR $<60 / \mathrm{ml} /$ $\min / 1.73 \mathrm{~m}^{2}$ ) were evaluated for cognitive dysfunction using the validated Standardized Mini-Mental State Examination (SMMSE). Individuals with dementia, depression or other psychiatric disorders were excluded, as were subjects on uric acid-lowering therapy or with serious illnesses such as severe anemia or active or ongoing cardiovascular or cerebrovascular disease. Results: 247 subjects were enrolled. SMMSE scores showed stepwise deterioration with increasing quartile of serum uric acid $(26.4 ; 26.1 ; 25.5 ; 25.3$, score range $20-$ $30, p=0.019)$. Post-hoc analysis demonstrated that there was no linear trend and only groups 1 and 4 were different with respect to SMMSE scores $(p=0.025)$. Stepwise multivariate linear regression revealed that age, educational status, pres-
\end{abstract}

\section{KARGER}

두 2011 S. Karger AG, Basel

Fax +41613061234 E-Mail karger@karger.ch www.karger.com www.karger.com/ajn ence of cerebrovascular disease, and serum uric acid were independently related to SMMSE scores. Conclusion: Serum uric acid levels are independently and inversely associated with mild cognitive dysfunction in subjects with CKD.

Copyright $\odot 2011$ S. Karger AG, Basel

\section{Introduction}

Chronic kidney disease (CKD) is an independent risk factor for cognitive dysfunction and dementia [1-4]. Cognitive decline in patients with kidney disease may be caused by various factors such as clinical and subclinical cerebrovascular disease and various comorbidities such as anemia, hypertension, diabetes, and malnutrition [5]. A better understanding of potential etiologies could lead to earlier interventions that might help reduce this important morbidity.

One of the most common causes of dementia is vascular cognitive impairment (VCI), which is strongly associated with chronic hypertension, cerebral microvascular disease, and the development of diffuse lesions of the white matter [6]. One of the key risk factors appears to be disease of the cerebral arterioles, which results in altered 
cerebral autoregulation that makes the distal cortex vulnerable to changes in blood pressure (BP), thereby increasing the risk to reduced cerebral pressure in the presence of low BP or to elevated cerebral pressure with sudden increases in systemic BP [6].

Hypertension is also associated with microvascular disease of the kidney (arteriolosclerosis), and experimental studies have also shown that its presence also alters renal autoregulation and increases the risk for renal progression (the Herrera hypothesis) [7, 8]. Since hypertension is associated with both renal and cerebral arteriolar disease, one might expect the two conditions to be commonly present in the same individual, thus providing a link between CKD and VCI. Indeed, in subjects with type 2 diabetes, the presence of cerebral microvascular disease (as manifested by silent cerebral infarction) is associated with increased risk for progression of renal disease [9].

While these studies implicate hypertension as the cause of arteriolar disease that then predisposes subjects to CKD and VCI, recent studies have suggested that elevated serum uric acid could also act as an etiologic factor [10]. Hyperuricemic rats develop renal microvascular disease involving the afferent arteriole that alters the autoregulatory response and results in glomerular hypertension $[11,12]$. In the remnant kidney model of CKD, the induction of hyperuricemia does not result in severe preglomerular arteriolar disease, and is associated with accelerated decline in renal function [13]. Importantly, the control of hypertension by thiazides in hyperuricemic rats does not block the development of renal arteriolopathy [14]. Rather, uric acid may directly induce vascular smooth muscle cell proliferation through a mechanism involving the stimulation of platelet-derived growth factor $[15,16]$.

Studies in humans have also linked uric acid with small vessel disease both in the kidney [17] and in the heart [18]. Elevated serum uric acid has been associated with increased risk for VCI and cerebral microvascular disease in the elderly [19-21]. Thus, we hypothesized that elevated uric acid might also be a risk for cognitive dysfunction in the CKD population. To test this hypothesis, we examined the relationship of serum uric acid with cognitive performance as determined using the Standardized Mini-Mental State Examination (SMMSE), which is a validated assessment tool for determining cognitive dysfunction $[22,23]$.

\section{Subjects and Methods}

\section{Study Population}

The current study was conducted in the outpatient nephrology unit of Zonguldak Ataturk State Hospital between May 2007 and
May 2010. The study was in accordance with the declaration of Helsinki, and informed consent was obtained from all patients before enrolment. The study population comprised patients with CKD defined as 24-hour creatinine clearance $<60 \mathrm{ml} / \mathrm{min} /$ $1.73 \mathrm{~m}^{2}$. Of 299 subjects that were screened for the study, 52 were excluded for the following prespecified reasons: unwillingness to participate in the study $(\mathrm{n}=16)$, dementia and Alzheimer disease (diagnosed by a neurologist; $\mathrm{n}=3$ ), use of antidepressants or depression (diagnosed by a psychiatrist; $n=14$ ), acute coronary syndrome (within the last 6 months; $n=4$ ), acute cerebrovascular disease (within the last 6 months; $\mathrm{n}=2$ ), active peripheral arterial disease $(n=1)$, hyper- or hypothyroidism $(n=4)$, severe anemia (defined as hemoglobin $<8 \mathrm{~g} / \mathrm{l} ; \mathrm{n}=2$ ), and 6 subjects (of whom 4 had a history of gout) who were actively using hypouricemic drugs. None of the patients reported any alcohol abuse. 247 subjects thus constituted the study population.

\section{Measurement of Cognitive Function}

The SMMSE was used to assess cognitive function and results in a score 30 (unimpaired) to 0 (impaired) [22]. It provides a global score of cognitive ability that correlates with function in activities of daily living. The SMMSE measures various domains of cognitive function including orientation to time and place, registration, concentration, short-term recall, naming familiar items, repeating a common expression, and the ability to read and follow written instructions, write a sentence, construct a diagram, and follow a three-step verbal command. The SMMSE takes approximately 10-15 min to administer, provides a baseline score of cognitive function and pinpoints specific deficits that can aid in forming a diagnosis. The SMMSE is a reliable instrument that allows practitioners to accurately measure cognitive deficits and deterioration over time [23]. The Turkish version of the SMMSE has been validated and shown to be reliable in the Turkish population [24]. Assistance was provided for patients who were illiterate. Informed consent was obtained from all participating patients. Permission for the study was obtained by the local ethics committee (Institutional Review Board).

\section{Additional Assessments}

Subjects with CKD also underwent the following assessments: medical history and physical examination, office BP measurements and 24-hour urinary creatinine clearance and albumin excretion. Sociodemographic and clinical assessment included age, gender, presence of diabetes and hypertension, alcohol use, smoking status, presence of coronary artery, cerebrovascular and peripheral artery diseases and current medications. Body mass index was calculated as the ratio of weight (in kilograms) to height squared (in square meters). Fasting blood samples were measured for serum hemoglobin, glucose, albumin, blood urea nitrogen, creatinine, uric acid, calcium, phosphorus, thyroid-stimulating hormone, total cholesterol, high-density lipoprotein cholesterol, low-density lipoprotein cholesterol, triglyceride, and high sensitive C-reactive protein.

\section{Office BP Measurements}

Office BP measurements were performed using a mercury sphygmomanometer. Adequately sized cuffs (standard cuff of 23 $\times 12 \mathrm{~cm}$ or a large cuff of $34 \times 15 \mathrm{~cm}$ ) according to arm circumference were placed on the nondominant arm. The first and fifth phases of Korotkoff sounds were taken as the systolic and dia- 
stolic BP, respectively. The measurements were taken after the patients had rested for $10 \mathrm{~min}$ in the sitting position with the arm comfortably placed at the heart level. Two measurements were taken at 5-min intervals. Each set of two measurements was averaged to give the office systolic and diastolic BPs. Clinical hypertension was defined as a $\mathrm{BP} \geq 140 / 90 \mathrm{~mm} \mathrm{Hg}$.

\section{Statistical Analyses}

Statistical analysis was performed using SPSS 15.0 for Windows (SPSS Inc., Chicago, Ill., USA). For the correlation of SMMSE score with various parameters Spearman and Pearson correlation coefficients were used as appropriate. Stepwise multivariate linear regression analyses were performed to assess the independent association of several variables with SMMSE score. The effects were measured by odds ratios and 95\% confidence intervals based on logistic regression models. Analysis of SMMSE scores according to uric acid quartiles was carried out by analysis of variance. For the post-hoc analysis of groups according to serum uric acid quartiles, Tukey's b test was used.

\section{Results}

The demographic characteristics, laboratory parameters, BP and SMMSE scores of the 247 enrolled subjects are shown in tables 1 and 2, respectively. Ninety-two subjects were hypertensive, and their treatment consisted of alpha-blockers $(n=29)$, beta-blockers $(n=75)$, calcium channel blockers $(\mathrm{n}=72)$, ACE inhibitors $(\mathrm{n}=49)$, angiotensin receptor blockers $(n=31)$, loop diuretics $(n=81)$ and thiazide diuretics combined with ACE inhibitors or angiotensin receptor blockers $(\mathrm{n}=46)$.

SSME scores ranged between 20 and 30 in all subjects, and showed a normal distribution. When SSME scores were categorized according to quartiles of serum uric acid, a progressive worsening of SSME scores were observed with increasing quartile of serum uric acid suggestive of worsening cognitive dysfunction (fig. $1 ; \mathrm{p}=0.019$ ). However, post-hoc analysis demonstrated that there was no linear trend, and only groups 1 and 4 were different with respect to SMMSE scores ( $\mathrm{p}=0.025)$.

By univariate analysis, SSMSE scores correlated with age (rho: $-0.337, \mathrm{p}<0.0001$ ), serum creatinine (rho: -0.132 , $\mathrm{p}=0.039$ ), blood urea nitrogen (rho: $-0.159, \mathrm{p}=0.012$ ), diastolic BP (rho: $+0.125, \mathrm{p}=0.01$ ) and serum uric acid (rho: $-0.261, p \leq 0.0001$ ). A weak but statistically significant relationship between uric acid (logarithmic values) and SMMSE score was observed (fig. 2; r = 0.297, p <0.001).

Stepwise multivariate linear regression analysis was performed to assess the independent effects of age, gender, smoking status, body mass index, systolic and diastolic BPs, educational status, presences of diabetes mellitus, coronary artery disease, cerebrovascular disease,
Table 1. Demographic characteristics and SMMSE scores of 247 chronic renal failure patients $(n=247$; mean $\pm S D)$

Age, years $60.5 \pm 11.0$

Males/females $118 / 129$

Illiterate and primary school graduate vs. other

$127 / 120$

Body mass index

Present/absent diabetes mellitus

$29.0 \pm 4.6$

$108 / 139$

Smokers/nonsmokers

$66 / 181$

Present/absent coronary artery disease $\quad 81 / 166$

Present/absent cerebrovascular disease 65/182

Present/absent peripheric artery disease 38/209

Present/absent hypertension

$155 / 92$

Systolic BP, mm Hg

$143.5 \pm 17.0$

Diastolic BP, mm Hg

$80.2 \pm 15.2$

$25.9 \pm 2.3$

Table 2. Laboratory parameters of 247 CKD patients

Hemoglobin, g/l

Blood glucose, $\mathrm{mmol} / \mathrm{l}$

Albumin, g/l

Blood urea nitrogen, $\mathrm{mmol} / \mathrm{l}$

Creatinine, $\mu \mathrm{mol} / \mathrm{l}$

Sodium, $\mathrm{mmol} / \mathrm{l}$

Potassium, $\mathrm{mmol} / \mathrm{l}$

Calcium, $\mathrm{mmol} / \mathrm{l}$

Phosphorus, $\mathrm{mmol} / \mathrm{l}$

Uric acid, $\mu \mathrm{mol} / \mathrm{l}$

High sensitive C-reactive protein, $\mathrm{mg} / \mathrm{dl}$

Total cholesterol, mmol/l

LDL-C, mmol/1

HDL-C, mmol/l

Triglyceride, $\mathrm{mmol} / \mathrm{l}$

Thyroid-stimulating hormone, $\mathrm{mU} / \mathrm{l}$

Creatinine clearance, $\mathrm{ml} / \mathrm{min}$

$$
\begin{gathered}
115.7 \pm 18.3 \\
8.8 \pm 4.0 \\
37.8 \pm 7.1 \\
14.3 \pm 7.2 \\
239.0 \pm 134.1 \\
138.4 \pm 4.2 \\
4.8 \pm 0.7 \\
2.25 \pm 0.16 \\
1.30 \pm 0.24 \\
455.6 \pm 115.8 \\
1.23 \pm 1.7 \\
4.97 \pm 3.6 \\
2.86 \pm 1.02 \\
1.04 \pm 0.32 \\
1.88 \pm 0.99 \\
1.87 \pm 1.36 \\
32.3 \pm 14.3
\end{gathered}
$$

Values are expressed as means \pm SD. LDL-C $=$ Low-density lipoprotein cholesterol; HDL-C = high-density lipoprotein cholesterol.

hemoglobin, thyroid-stimulating hormone level, 24-hour creatinine clearance and albumin excretion, serum uric acid, high sensitive C-reactive protein and use of alphablockers, beta-blockers, calcium channel blockers, ACE inhibitors, angiotensin receptor blockers, loop diuretics and thiazide diuretics combined with ACE inhibitors or angiotensin receptor blockers with SMMSE score. Age, educational status, presence of cerebrovascular disease, and serum uric acid were found to be independently related to SMMSE scores (table 3). 


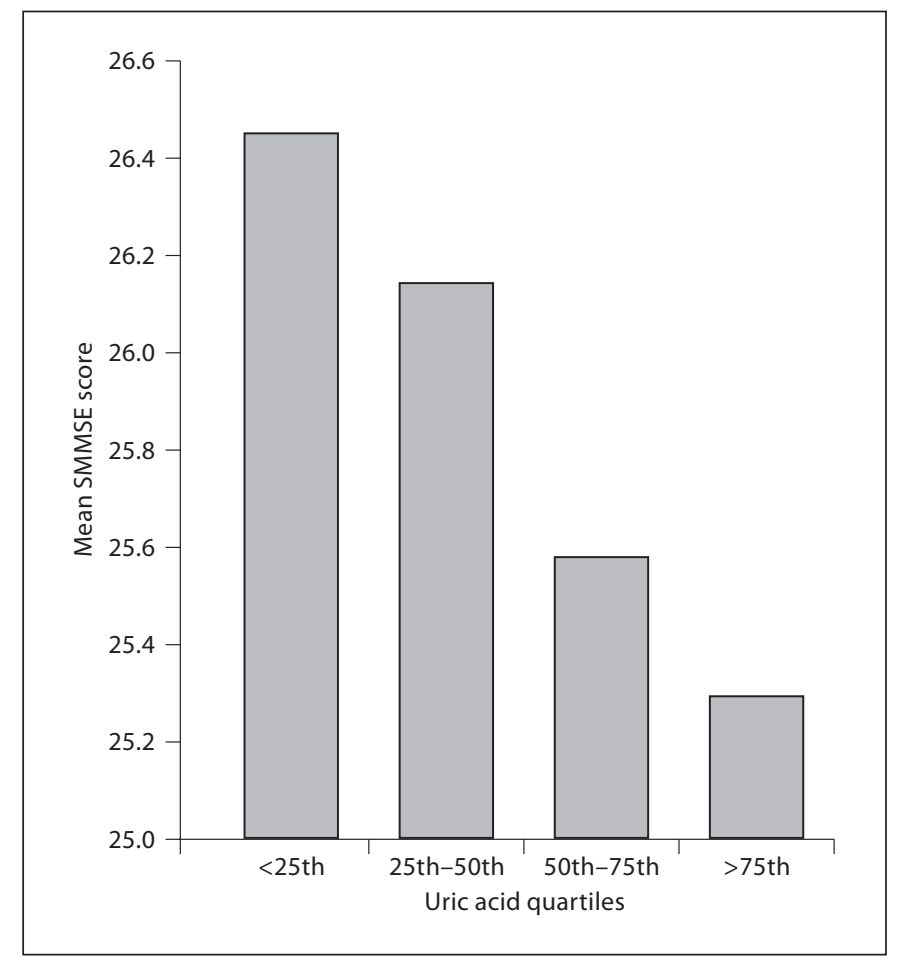

Fig. 1. Mean SMMSE scores according to uric acid quartiles. Subjects were divided into 4 groups based on uric acid quartiles: $<25$ th quartile (58 patients): uric acid levels $<380.7 \mu \mathrm{mol} / \mathrm{l} ; 25$ th50th quartile (63 patients): uric acid levels 380.7-434.8 $\mu \mathrm{mol} / \mathrm{l}$; 50th-75th quartile (64 patients): uric acid level 434.8-517.5 $\mu \mathrm{mol} / \mathrm{l}$, and $>75$ th quartile (62 patients): uric acid level $>517.5$ $\mu \mathrm{mol} / \mathrm{l}(\mathrm{p}=0.019)$.

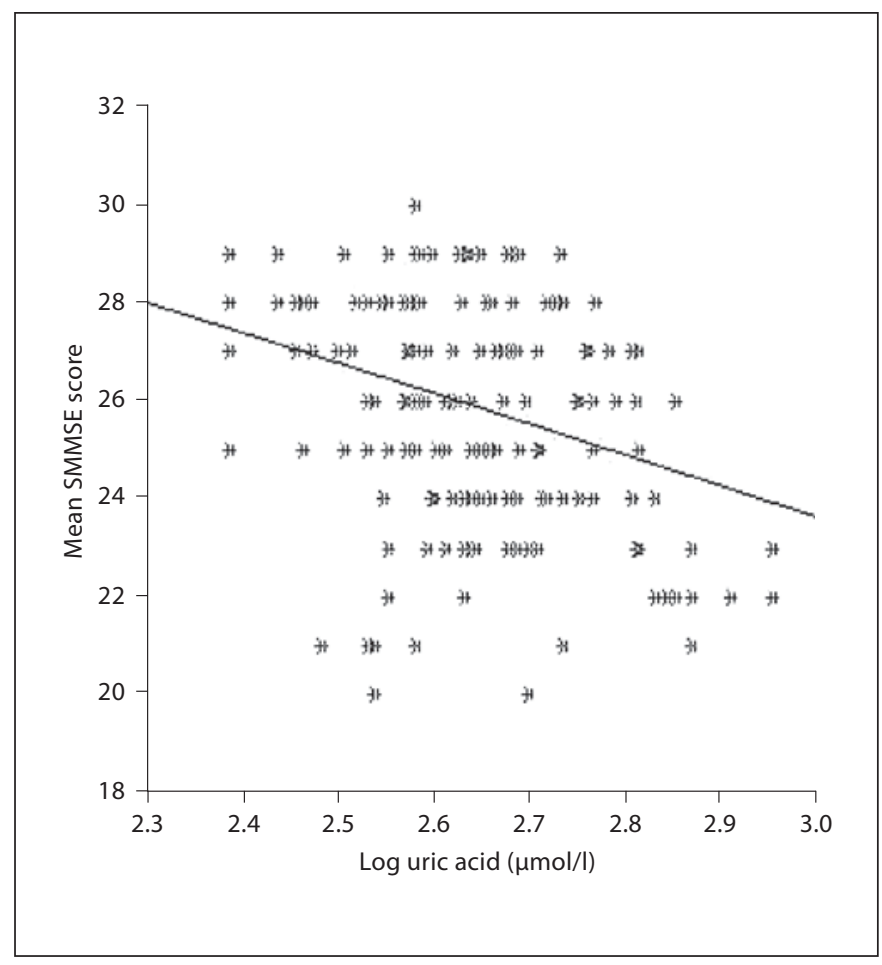

Fig. 2. Regression graphic for logarithmically converted uric acid levels and SMMSE score $(\mathrm{r}=-0.297, \mathrm{p}<0.0001)$.

Table 3. Linear regression analysis of factors related to SMMSE scores

\begin{tabular}{lccc}
\hline Parameter & B & $95 \%$ CI & p \\
\hline Educational status (illiterate and & & & \\
$\quad$ elementary school vs. higher educational level) & -1.427 & -2.004 to -0.851 & $<0.0001$ \\
Age & -0.030 & -0.059 to -0.002 & 0.039 \\
Presence of cerebrovascular disease & -1.341 & -1.940 to -0.742 & $<0.0001$ \\
Serum uric acid & -0.005 & -0.007 to -0.002 & $<0.0001$ \\
\hline
\end{tabular}

\section{Discussion}

In this study, we performed a simple cognitive function test (SMMSE) in subjects with CKD, and used this to identify risk factors for cognitive dysfunction. Univariate analysis identified diastolic BP, renal function (creatinine clearance and 24-hour urinary albumin excretion), and serum uric acid as risk factors. By multi- variate analysis, age, educational level, presence of cerebrovascular disease, and serum uric acid levels remained significant predictors.

The major new finding is that elevated serum uric acid was independently associated with worse cognitive function in subjects with CKD. Our data are consistent with previous reports - mainly from healthy elderly - suggesting that serum uric acid predicts cognitive decline and 
white matter lesions are consistent with cerebral ischemia $[19,21]$. Elevated serum uric acid has also been shown to predict both hypertension and stroke by meta-analyses $[25,26]$. The observation that elevated uric acid can also induce vascular smooth muscle cell proliferation in vitro $[14,15]$ and induce renal microvascular disease in vivo $[12,27]$ suggests that it may also be able to induce cerebral microvascular disease, which underlies the development of VCI [28].

Of note, there are some contradictory data. Serum uric acid levels tend to be low in subjects with established Alzheimer's dementia or VCI [29-31]. Some studies also suggest that elevated uric acid might reduce the risk for progression of dementia in subjects with underlying impaired cognitive function [32]. One study reported that the risk for developing dementia in subjects with a high uric acid is due to the frequent coexistence of hypertension and cardiovascular disease, and in the absence of such associated risk factors, elevated uric acid may actually reduce the risk for dementia [33].

Several potential mechanisms might account for such an apparent paradox. For example, since serum uric acid reflects to some extent the nutritional status, a lower uric acid could reflect a reduction in intake as may be observed in subjects with progressive dementia. In dialysis patients, for example, lower uric acid levels are associated with low serum albumin, a bedridden state, or a history of cerebrovascular accident [34]. A lower serum uric acid may also reflect a reduction in serum antioxidants, which has been postulated to increase the risk for Alzheimer's dementia [29-31]. This possibility is based on studies that show that uric acid is a major antioxidant in human plasma and can scavenge numerous oxidants in vitro, including superoxide, hydroxyl radical and peroxynitrite $[35,36]$. However, when uric acid reacts with peroxynitrite, it will also generate free radicals in the process, including triuretcarbonyl and aminocarbonyl radicals [37]. Furthermore, while uric acid is an antioxidant in the extracellular environment, inside the cell it induces endothelial dysfunction [38], oxidative stress [39-41], inflammation [42, 43] and stimulation of vasoactive mediators such as thromboxane and angiotensin II $[13,39,40]$. Thus, it remains possible that uric acid might have both deleterious and beneficial effects on neural function. Nevertheless, our studies suggest that in CKD the presence of elevated uric acid is associated with worse cognitive function. Furthermore, pilot studies suggest that lowering uric acid in subjects with CKD may have beneficial effects on renal function, BP, and inflammation, and in one study it was associated with a marked (70\%) reduction in cardiovascular events [44-46].
Our study has limitations. First, our study is cross-sectional, and hence cause and effect can not be ascertained. Second, the overall impact of elevated uric acid on cognitive function was relatively weak, even though it was statistically significant. Third, we did not perform computed tomography or MRI in our patients to determine a relationship of uric acid with white matter lesions such as observed in subjects with VCI. Thus, we can not rule out the possibility of subtle/silent cerebral lesions. Fourth, the SMMSE is not the gold standard for the detection of cognitive dysfunction; however, it is easy to administer and provides a global score of cognitive ability that correlates with function in activities of daily living [23].

In conclusion, one of the increasingly recognized complications of CKD is cognitive decline [1-3]. In this paper, we presented the first epidemiological evidence that this decline correlates with increased serum uric acid levels. If uric acid has a causal role in VCI via its ability to cause microvascular disease and hypertension, then this could be a significant problem in subjects with CKD as hyperuricemia occurs in approximately half of the subjects by the time the dialysis is initiated [34, 47]. We believe that extending these cross-sectional findings to longitudinal studies would be helpful to determine whether elevated uric acid increases the risk or rate of cognitive decline in CKD patients.

\section{Acknowledgement}

Support for this paper was provided in part by NIH grant HL68607

\section{Disclosure Statement}

Dr. Johnson has several patent applications related to the lowering of uric acid as a means for preventing metabolic syndrome and hypertension.

References

1 Kurella M, Chertow GM, Fried LF, et al: Chronic kidney disease and cognitive impairment in the elderly: the health, aging, and body composition study. J Am Soc Nephrol 2005; 16:2127-2133.

-2 Kurella M, Yaffe K, Shlipak MG, Wenger NK and Chertow GM: Chronic kidney disease and cognitive impairment in menopausal women. Am J Kidney Dis 2005;45:66-76.

3 Kurella Tamura M, Wadley V, Yaffe K, et al: Kidney function and cognitive impairment in US adults: the Reasons for Geographic and Racial Differences in Stroke (REGARDS) Study. Am J Kidney Dis 2008;52:227-234. 
-4 Seliger SL, Siscovick DS, Stehman-Breen $\mathrm{CO}$, et al: Moderate renal impairment and risk of dementia among older adults: the Cardiovascular Health Cognition Study. J Am Soc Nephrol 2004;15:1904-1911.

$\checkmark 5$ Pereira AA, Weiner DE, Scott T, Sarnak MJ: Cognitive function in dialysis patients. Am J Kidney Dis 2005;45:448-462.

6 Cherubini A, Lowenthal DT, Paran E, Mecocci P, Williams LS, Senin U: Hypertension and cognitive function in the elderly. Am J Ther 2007; 14:533-554.

7 Johnson RJ, Segal MS, Srinivas T, et al: Essential hypertension, progressive renal disease, and uric acid: a pathogenetic link? J Am Soc Nephrol 2005; 16:1909-1919.

$\checkmark 8$ Sanchez-Lozada LG, Tapia E, Johnson RJ, Rodriguez-Iturbe B, Herrera-Acosta J: Glomerular hemodynamic changes associated with arteriolar lesions and tubulointerstitial inflammation. Kidney Int Suppl 2003; 86:S9-S14.

9 Uzu T, Kida Y, Shirahashi N, et al: Cerebral microvascular disease predicts renal failure in type 2 diabetes. J Am Soc Nephrol 2010;21: 520-526.

-10 Kanbay M, Sanchez-Lozada LG, Franco M, et al: Microvascular disease and its role in the brain and cardiovascular system: a potential role for uric acid as a cardiorenal toxin. Nephrol Dial Transplant 2011;26:430-437.

- 11 Sanchez-Lozada LG, Tapia E, RodriguezIturbe $\mathrm{B}$, Johnson RJ, Herrera-Acosta J: Hemodynamics of hyperuricemia. Semin Nephrol 2005;25:19-24.

-12 Sanchez-Lozada LG, Tapia E, Santamaria J, et al: Mild hyperuricemia induces vasoconstriction and maintains glomerular hypertension in normal and remnant kidney rats. Kidney Int 2005;67:237-247.

$\checkmark 13$ Kang DH, Nakagawa T, Feng L, et al: A role for uric acid in the progression of renal disease. J Am Soc Nephrol 2002;13:2888-2897.

14 Mazzali M, Kanellis J, Han L, et al: Hyperuricemia induces a primary renal arteriolopathy in rats by a blood pressure-independent mechanism. Am J Physiol 2002;282:F991F997.

$\checkmark 15$ Rao GN, Corson MA, Berk BC: Uric acid stimulates vascular smooth muscle cell proliferation by increasing platelet-derived growth factor A-chain expression. J Biol Chem 1991;266:8604-8608.

$>16$ Watanabe S, Kang DH, Feng L, et al: Uric acid, hominoid evolution, and the pathogenesis of salt-sensitivity. Hypertension 2002; 40:355-360.

17 Wu J, Chen X, Xie Y, et al: Characteristics and risk factors of intrarenal arterial lesions in patients with IgA nephropathy. Nephrol Dial Transplant 2005;20:719-727.

18 Basara N, Kanbayb M, Sen N, et al: Elevated serum uric acid predicts angiographic impaired reperfusion and one-year mortality in ST-segment elevation myocardial infarction patients undergoing percutaneous coronary intervention. J Investig Med, in press.
19 Schretlen DJ, Inscore AB, Jinnah HA, Rao V, Gordon B, Pearlson GD: Serum uric acid and cognitive function in community-dwelling older adults. Neuropsychology 2007;21:136140.

20 Schretlen DJ, Inscore AB, Vannorsdall TD, et al: Serum uric acid and brain ischemia in normal elderly adults. Neurology 2007;69: 1418-1423.

21 Vannorsdall TD, Jinnah HA, Gordon B, Kraut M, Schretlen DJ: Cerebral ischemia mediates the effect of serum uric acid on cognitive function. Stroke 2008;39:3418-3420.

22 Molloy DW, Standish TI: A guide to the standardized Mini-Mental State Examination. Int Psychogeriatr 1997;9(suppl 1):87-94, discussion 143-150.

23 Vertesi A, Lever JA, Molloy DW, et al: Standardized Mini-Mental State Examination. Use and interpretation. Can Fam Physician 2001;47:2018-2023.

24 Güngen C, Ertan T, Eker E, Yaşar R, Engin F: Reliability and validity of the standardized Mini Mental State Examination in the diagnosis of mild dementia in the Turkish population. 2002;13:273-281 (in Turkish).

25 Kim SY, Guevara JP, Kim KM, Choi HK, Heitjan DF, Albert DA: Hyperuricemia and risk of stroke: a systematic review and metaanalysis. Arthritis Rheum 2009;61:885-892.

26 Grayson PC, Kim SY, Lavalley M, Choi HK: Hyperuricemia and incident hypertension: a systematic review and meta-analysis. Arthritis Care Res (Hoboken) 2011;63:102-110.

27 Sanchez-Lozada LG, Tapia E, Soto V, et al: Effect of febuxostat on the progression of renal disease in 5/6 nephrectomy rats with and without hyperuricemia. Nephron Physiol 2008;108:69-78.

28 Cherubini A, Lowenthal DT, Paran E, Mecocci P, Williams LS, Senin U: Hypertension and cognitive function in the elderly. Dis Mon 2010;56:106-147.

29 Kim TS, Pae CU, Yoon SJ, et al: Decreased plasma antioxidants in patients with Alzheimer's disease. Int J Geriatr Psychiatry 2006;21:344-348.

30 Rinaldi P, Polidori MC, Metastasio A, et al: Plasma antioxidants are similarly depleted in mild cognitive impairment and in $\mathrm{Alz}$ heimer's disease. Neurobiol Aging 2003;24: 915-919.

-31 Polidori MC, Mattioli P, Aldred S, et al: Plasma antioxidant status, immunoglobulin $g$ oxidation and lipid peroxidation in demented patients: relevance to Alzheimer disease and vascular dementia. Dement Geriatr Cogn Disord 2004;18:265-270.

-32 Irizarry MC, Raman R, Schwarzschild MA, et al: Plasma urate and progression of mild cognitive impairment. Neurodegener Dis 2009;6:23-28.

33 Euser SM, Hofman A, Westendorp RG, Breteler MM: Serum uric acid and cognitive function and dementia. Brain 2009;132:377382.

34 Lee SM, Lee AL, Winters TJ, et al: Low serum uric acid level is a risk factor for death in incident hemodialysis patients. Am J Nephrol 2009;29:79-85.
35 Ames BN, Cathcart R, Schwiers E, Hochstein P: Uric acid provides an antioxidant defense in humans against oxidant- and radicalcaused aging and cancer: a hypothesis. Proc Natl Acad Sci U S A 1981;78:6858-6862.

36 Becker BF, Reinholz N, Leipert B, Raschke P, Permanetter B, Gerlach E: Role of uric acid as an endogenous radical scavenger and antioxidant. Chest 1991;100:176S-181S.

>37 Imaram W, Gersch C, Kim KM, Johnson RJ, Henderson GN, Angerhofer A: Radicals in the reaction between peroxynitrite and uric acid identified by electron spin resonance spectroscopy and liquid chromatography mass spectrometry. Free Radic Biol Med 2010;49:275-281.

38 Khosla UM, Zharikov S, Finch JL, et al: Hyperuricemia induces endothelial dysfunction. Kidney Int 2005;67:1739-1742.

-39 Corry DB, Eslami P, Yamamoto K, Nyby MD, Makino H, Tuck ML: Uric acid stimulates vascular smooth muscle cell proliferation and oxidative stress via the vascular renin-angiotensin system. J Hypertens 2008; 26:269-275.

40 Yu MA, Sanchez-Lozada LG, Johnson RJ, Kang DH: Oxidative stress with an activation of the renin-angiotensin system in human vascular endothelial cells as a novel mechanism of uric acid-induced endothelial dysfunction. J Hypertens 2010;28:12341242.

41 Sanchez-Lozada LG, Soto V, Tapia E, et al: Role of oxidative stress in the renal abnormalities induced by experimental hyperuricemia. Am J Physiol 2008;295:F1134-F1141.

42 Kanellis J, Watanabe S, Li JH, et al: Uric acid stimulates monocyte chemoattractant protein-1 production in vascular smooth muscle cells via mitogen-activated protein kinase and cyclooxygenase-2. Hypertension 2003; 41:1287-1293.

43 Kang DH, Park SK, Lee IK, Johnson RJ: Uric acid-induced C-reactive protein expression: implication on cell proliferation and nitric oxide production of human vascular cells. J Am Soc Nephrol 2005; 16:3553-3562.

44 Siu YP, Leung KT, Tong MK, Kwan TH: Use of allopurinol in slowing the progression of renal disease through its ability to lower serum uric acid level. Am J Kidney Dis 2006; 47:51-59.

45 Talaat KM, El-Sheikh AR: The effect of mild hyperuricemia on urinary transforming growth factor beta and the progression of chronic kidney disease. Am J Nephrol 2007; 27:435-440.

46 Goicoechea M, de Vinuesa SG, Verdalles U, et al: Effect of allopurinol in chronic kidney disease progression and cardiovascular risk. Clin J Am Soc Nephrol 2010;5:1388-1393.

-47 Suliman ME, Johnson RJ, Garcia-Lopez E, et al: J-shaped mortality relationship for uric acid in CKD. Am J Kidney Dis 2006;48:761771 . 